Background Although minority HIV-1 drug-resistant HIV-1 variants may be selected under antiretroviral pressure, leading to therapy failure, their clinical significance remains controversial. This is particularly relevant in the case of prevention of mother-to-child transmission (MTCT), where transmitted drug resistance can affect treatment outcomes.

Methods An ultrasensitive HIV-1 genotyping assay based on deep sequencing (DEEPGENHIV) with a $1 \%$ mutation frequency sensitivity, was used to quantify MTCT drug-resistant variants in 38 prenatally HIV-infected children experiencing (Group I, $n=27$ ) or not (Group II, $n=11$ ) virologic failure 12 months after initiating first-line antiretroviral therapy (ART) as part of a paediatric cohort in Uganda.

Results Infants were infected with subtype $A(n=20), D(n=16)$ or $\mathrm{C}(\mathrm{n}=2)$ HIV-1 strains, distributed equally between both patients' groups. Similarly, no significant difference was observed in intra-patient HIV-1 diversity among viruses obtained from Group I or II individuals at baseline. DEEPGENHIV was able to detect all the mutations originally detected in samples obtained from four control patients in Group II, where drug resistance was identified at baseline using Sanger sequencing, e.g. K65R (78\% mutation frequency), K103N (47\%), or M184V (85\%). More importantly, a series of low abundance ( $<20 \%$ detection limit of Sanger) primary and compensatory mutations associated with resistance to PIs (D30N, Q48V), NRTIs (D67N, K219Q), or NNRTIs (L100I, $\mathrm{K} 103 \mathrm{~N})$ were identified in both groups of patients, although just a few seem to have been selected and became majority variants after 12 or 24 months of ART.

Conclusion DEEPGENHIV improves the detection of minority viral variants in infants following MTCT; however, most of the emergent HIV-1 drug resistance mutations were not present at low frequency at baseline in subjects failing ART, most likely being generated and selected following exposure to treatment. Further studies, using this or other ultrasensitive assays, are needed to better understand the transmission, dynamics and overall evolution of minority drug-resistant viruses in MTCT.

\section{OC 8453 SCALING-UP MASS TESTING AND TREATMENT EFFECTIVELY REDUCED MALARIA ASYMPTOMATIC PARASITAEMIA IN CHILDREN UNDER 15 YEARS OF AGE IN GHANA}

${ }^{1}$ Ignatius Cheng Ndong* ${ }^{1}$ Collins Stephan Ahorlu, ${ }^{1}$ Daniel Okyere ${ }^{1}$ Alexander A Nyarko, ${ }^{2}$ Alfred Amambua-Ngwa, 'Kwakwo A Koram. 'Noguchi Memorial Institute for Medical Research, University of Ghana, Accra, Ghana; ${ }^{2}$ Medical Research Council, Serrekunda, The Gambia

\subsection{6/bmjgh-2019-EDC.16}

Background Global efforts to scale up malaria control interventions that work, are gaining momentum. These include the use of long-lasting insecticide nets, intermittent preventive treatment and test, treat and track using artemisinin-based combination therapies. Mass testing, treatment and tracking (MTTT) of the entire populations complemented by homebased management (HBM) using volunteers could be an effective approach at lower cost. MTTT of children in Ghana has demonstrated a parasite load reduction of 90\%. However, unanswered questions include - Could this be scaled-up? What proportion of the community could be covered? What do we need? Can MTTT reduce asymptomatic parasitaemia levels in under-15 children?
Methods The target population for this study was 5000 asymptomatic individuals in seven communities in the Pakro subdistrict in Ghana. A community register was developed following a census. Community volunteers conducted quarterly house-to-house testing (using RDTs) and treating positive cases with ACTs. Between interventions HBM was conducted.

Results In those tested, asymptomatic malaria parasitaemia reduced from 1795 (36.3\%) in July 2017 to 942 (23.1\%) in March 2018. In eight months, parasitaemia declined by $43.5 \%$ and $37.3 \%$ in children under 15 and under 5, respectively. Coverage was $98.8 \%$ in July 2017 and $81.4 \%$ in March 2018. One of the challenges that surfaced was the fact that decrease in hospital attendance had a negative effect on money generated by the health facility. The district and subdistrict services, though appreciating the work, indicated that the negative effect on the health system may be serious and that measures need to be taken to address alternative financing for the health system.

Conclusion This study has demonstrated that combining MTTT and home-based management of malaria could reduce prevalence in under-15 children and that using community volunteers could ensure effective coverage at lower cost. There is need to start looking at financing of the health system without malaria.

\section{OC 8459 ASSESSMENT OF PARASITE CLEARANCE AFTER REPEATED TREATMENT WITH ARTESUNATE AMODIAQUINE, DIHYDROARTEMISININ-PIPERAQUINE, PYRONARIDINE-ARTESUNATE IN MALARIA PATIENTS IN BURKINA FASO}

Issiaka Soulama*, Sodiomon B Sirima. Centre National de Recherche et de Formation sur le Paludisme, Ouagadougou, Burkina Faso

\subsection{6/bmjgh-2019-EDC.17}

Background Reports from Southeast Asia showed delayed parasite clearance after treatment with known artemisinin-based combination therapies (ACTs), the first-line treatment for malaria. We then carried out a study in the framework of the WANECAM clinical trial to assess comparatively the parasite clearance time and rate from $P$. falciparum malaria patients repeatedly treated with the artesunate-amodiaquine (ASAQ), dihydroartemisinin-piperaquine (DHA-PQ) and artesunate-pyronaridine (PYR).

Methods A randomised, phase III/IV comparative, multicentre, open-label, parallel 3-arms trial was conducted in Banfora Health District area comparing the efficacy of a three-day regimen of DHA-PQ, PYR with ASAQ for the treatment of children (above 6 months) and adults with uncomplicated falciparum malaria. From August 2012 to December 2013, each randomised patient was followed up for 42 days over a period of two years. Treatment was directly observed, and blood smear samples were collected twice daily (12 hour \pm 2 hour) until parasite clearance.

The endpoints of the present sub-study were parasite clearance rate and time. The secondary endpoints included PCRcorrected and uncorrected cure rates.

Results Out of 2843 screened patients, 763 were recruited for parasite clearance endpoint analyses. The median parasite clearance time (PCT) was 24.1 hour (2-sided 95\% CI, 24.0 to 24.2 hour), 23.9 hour (2-sided 95\% CI, 23.8 to 24.0 hour) and 24.2 hour (2-sided 95\% CI, 24.1 to 24.5 hour), in PYR 